\title{
Prognostic Value of Endotoxemia in Patients with Gram-Negative Bacteremia Is Bacterial Species Dependent
}

\author{
James C. Hurley ${ }^{a-c}$ Steven M. Opal ${ }^{d}$ \\ ${ }^{a}$ Rural Health Academic Center, Melbourne Medical School, University of Melbourne, ${ }^{b}$ Infection Control Committees, \\ Ballarat Health Services and St John of God Hospital, and ' Division of Internal Medicine, Ballarat Health Services, \\ Ballarat, Vic., Australia; ${ }^{~ I n f e c t i o u s ~ D i s e a s e ~ D i v i s i o n, ~ A l p e r t ~ M e d i c a l ~ S c h o o l ~ o f ~ B r o w n ~ U n i v e r s i t y, ~ P r o v i d e n c e, ~ R . I ., ~ U S A ~}$
}

\author{
Key Words \\ Endotoxemia - Gram-negative bacteremia - Escherichia coli . \\ Sepsis · Septic shock · Limulus assay
}

\begin{abstract}
The prognostic impact of endotoxemia detection in sepsis is unclear. Endotoxemia is detectable in $<70 \%$ of patients with Gram-negative (GN) bacteremias. Mortality proportion data were available from 27 published studies of patients with GN bacteremia in various settings. Among ten studies restricted to specific types of GN bacteremia, endotoxemia was associated with significantly increased mortality risk for Neisseria meningitidis (4 studies; 138 bacteremias; OR $26.0 ; 95 \% \mathrm{Cl}$, 1.6-321) but not for Salmonella enterica serovar Typhi (3 studies; 36 bacteremias; OR 0.89; $95 \% \mathrm{Cl}, 0.01-74.1)$. For 17 unrestricted studies ( $319 \mathrm{GN}$ bacteremic patients), endotoxemia was associated with an increased mortality risk with non-Escherichia coli Enterobacteriaceae such as Klebsiella and Enterobacter species (97 bacteremias; OR 3.7; 95\% Cl, $1.3-10.3$ ). By contrast, E. coli (144 bacteremias; OR 0.78; $95 \%$ $\mathrm{Cl}, 0.36-1.7)$, and non-Enterobacteriaceae species such as Pseudomonas species (78 bacteremias; OR $1.7 ; 95 \% \mathrm{Cl}$, 0.74.6) had no increased mortality risk. That endotoxemia de-
\end{abstract}

\section{KARGER}

E-Mail karger@karger.com

www.karger.com/jin tection is predictive of mortality among patients bacteremic with non-E. coli Enterobacteriaceae but not E. coli is surprising given the presumed commonality of the hexa-acyl lipid A structure among Enterobacteriaceae species.

Copyright $\odot 2013$ S. Karger AG, Basel

\section{Introduction}

There is extensive evidence implicating endotoxin in the pathogenesis of Gram-negative (GN) sepsis [1, 2]. Hence, an assay to detect endotoxemia would appear to be a logical adjunct toward targeting novel therapies for sepsis including those specifically directed against endotoxin [3].

However, among large clinical studies, the predictive value of endotoxemia detection remains unclear [4] and a disconnect with animal models exists $[5,6]$. On the one hand, studies of patients with plague $[7,8]$, or meningococcemia [9], have found a quantitative correlation between the level of endotoxemia and the degree of organ dysfunction or mortality. On the other hand, larger studies of sepsis in which the type of GN bacteremias were not restricted to any specific GN species type [10-20], have
(C) 2013 S. Karger AG, Basel

$1662-811 \mathrm{X} / 13 / 0056-0555 \$ 38.00 / 0$ 
generated findings that were contradictory. In aggregate, the results from these studies indicate that endotoxemia detection is a borderline predictor of mortality risk (odds ratio $<2)[4,21]$.

These and some experimental observations [22, 23] raise doubt as to whether the relationship between endotoxemia and outcome is equal for GN bacteremias of different species type. Clarification might also help to reconcile conflicting observations in the clinical literature especially given the variable prevalence of different GN bacteremia species types in different studies [4].

The objective here is to re-examine the prognostic value of endotoxemia in association with bacteremias with Escherichia coli versus other species of GN bacteria in the light of four observations. Firstly, mortality occurs less frequently for $E$. coli bacteremias versus other types of GN bacteremias [24-26]. Second, there are specific structural differences in lipid A, the biologically active component of endotoxin (lipopolysaccharide, LPS) for Enterobacteriaceae including $E$. coli versus other GN bacteria such as non-Enterobacteriaceae [1]. There are important discrepancies between the in vivo biological activities of the endotoxins of different types of GN bacteria versus their activities in the limulus assay, which is the most commonly used assay for endotoxemia detection $[22,27,28]$. Finally, endotoxemia is undetectable for $>20 \%$ of GN bacteremias overall and $>40 \%$ of E. coli [29] bacteremias.

\section{Methods}

\section{Data Sources}

A computerized search of PubMed (including Medline) was undertaken using the key words 'endotoxemia' and 'limulus' in the title or abstract and was restricted to studies in humans. This search was supplemented by a hand search for studies reporting mortality outcome data in relation to endotoxemia detection and detection of GN bacteremia with blood culture for patient groups at risk of GN bacteremia. This search has been performed and repeatedly updated [21,29] between 1993 and April 2012 as detailed previously [4] and a call for data was issued [30]. Additional data was obtained through correspondence with authors of potentially eligible studies.

\section{Study Selection}

The study inclusion criteria was as detailed previously [4] with the following additional criteria: (1) at least 5 patients with GN bacteremia, and (2) survival outcome available on an individual patient basis in relation to endotoxemia detection and type of GN bacteremia. Additional information was received by personal communication for 11 studies.

The studies were classified as to whether they were not restricted to examining specific GN bacteremias (unrestricted studies), versus studies that were so restricted (restricted studies) to a single GN pathogen type (e.g., Salmonella typhi, Neisseria meningitidis, Yersinia pestis, and Burkholderia pseudomallei). For the unrestricted studies, the individual patients with mono-microbial GN bacteremias were classified into four groups as follows: (1) GN bacteremias with E. coli, (2) GN bacteremias with Enterobacteriaceae other than E. coli (e.g., Klebsiella pneumoniae, Enterobacter species, Serratia marcescens, Proteus mirabilis, and Providencia rettgeri), (3) GN bacteremias other than Enterobacteriaceae (e.g., Pseudomonas species, Acinetobacter species, and Bacteroides species). According to the classification by Munford [1], the GN bacteria of the first two groups are known to have a hexa-acyl lipid A whereas those of the third group usually have a lipid A that is either non-hexa-acyl or of unknown structure. The following GN bacteremias were not included in this analysis due to small numbers and uncertainties in classification: Campylobacter species, Neisseria gonnorhoeae, non-typhi Salmonellae, Haemophilus influenzae.

Data Extraction and Analysis: Calculation of an Odds Ratio

The endotoxemia detection and mortality outcome data for all studies was extracted for those with GN bacteremia on a per patient basis. The determination of endotoxemia present versus absent was as recorded in the original publication without any attempt to standardize endotoxemia detection levels across publications. An odds ratio (OR) was then calculated as follows:

$\mathrm{OR}=$ (endotoxemia positive with fatal outcome/endotoxemia positive with non-fatal outcome)/(endotoxemia negative with fatal outcome/endotoxemia negative with non-fatal outcome).

For the purposes of graphical representation, a forest plot was generated using summary OR and 95\% confidence intervals (CI) as derived using The Mantel-Haenszel method in which 0.5 was added to any cell containing a zero to avoid deriving ORs that were undefined. These calculations and graphics were undertaken using the 'Metan' command [31] in STATA (release 11.0; STATA Corp., College Station, Tex., USA). This enables both a visual and statistical evaluation of homogeneity [32].

There exists within the dataset the following data issues: small samples, sparse data and zero cells. Hence, two different methods of statistical analysis were undertaken. First, to allow for these issues summary level ORs and 95\% CIs for each category of GN bacteremia were derived by exact grouped logistic regression (ELR) methods using the 'exlogistic' command in STATA.

Second, because the mortality proportions had been derived from clusters of patients within studies, the independence of the data is not a tenable assumption. This assumption is vitiated by the potentially infectious nature of some of the GN bacteremias under study. Hence, for the purpose of comparisons across categories of GN bacteremias and to derive a test for interaction between the detection of endotoxemia and the type of GN bacteremia, the method of generalized estimating equations (GEE) was used [33].

\section{Results}

There were 17 studies that were not restricted (table 1) and 10 studies that were restricted (table 2) to a single GN pathogen type. 
Table 1. Mortality proportions for patients with GN bacteremia (unrestricted studies)

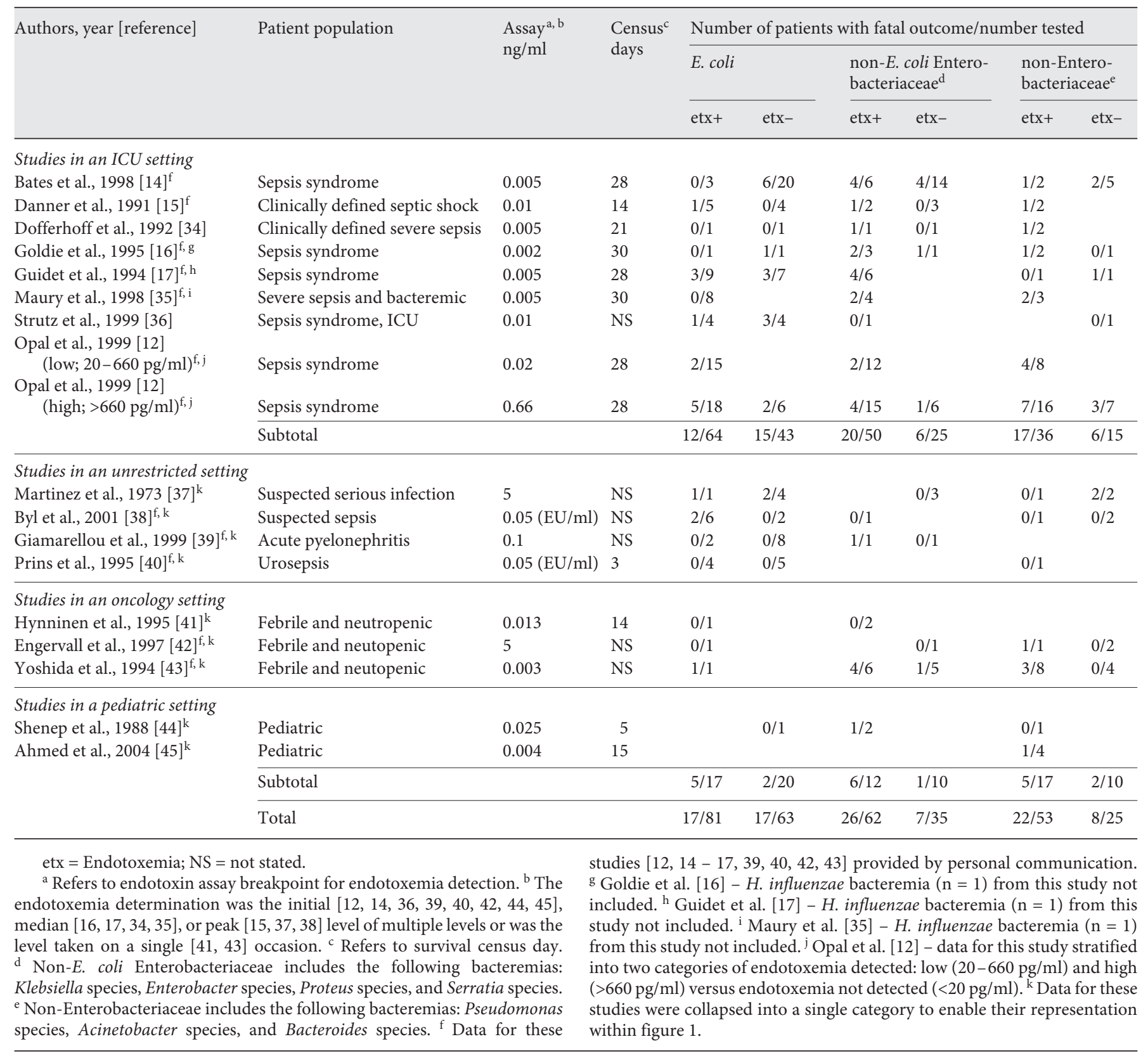

\section{Unrestricted Studies}

Eight unrestricted studies $[12,14-17,34-36]$ undertaken in an ICU setting were identified reporting the results for a total of $233 \mathrm{GN}$ bacteremic patients of which $76(33 \%)$ had a fatal outcome (table 1). In these studies $E$. coli was the most common GN bacteremia and had the least mortality (107 with 27 deaths; $25 \%$ ), versus non-E. coli Enterobacteriaceae (75 with 26 deaths; $35 \%$ ) and ver- sus non-Enterobacteriaceae (51 with 23 deaths; 45\%). There were an additional 9 non-ICU studies in which there were $86 \mathrm{GN}$ bacteremias in the three categories with 21 fatalities (24\%). Among these 9 unrestricted non-ICU studies are 4 studies limited to adult patients [37-40], 3 studies of febrile oncology patients [41-43] and 2 studies limited to a pediatric age group $[44,45]$ (table 1$)$. In only 3 studies [38-40] was it explicitly stated that endotoxemia 
Table 2. Mortality proportions for patients with GN bacteremia (restricted studies)

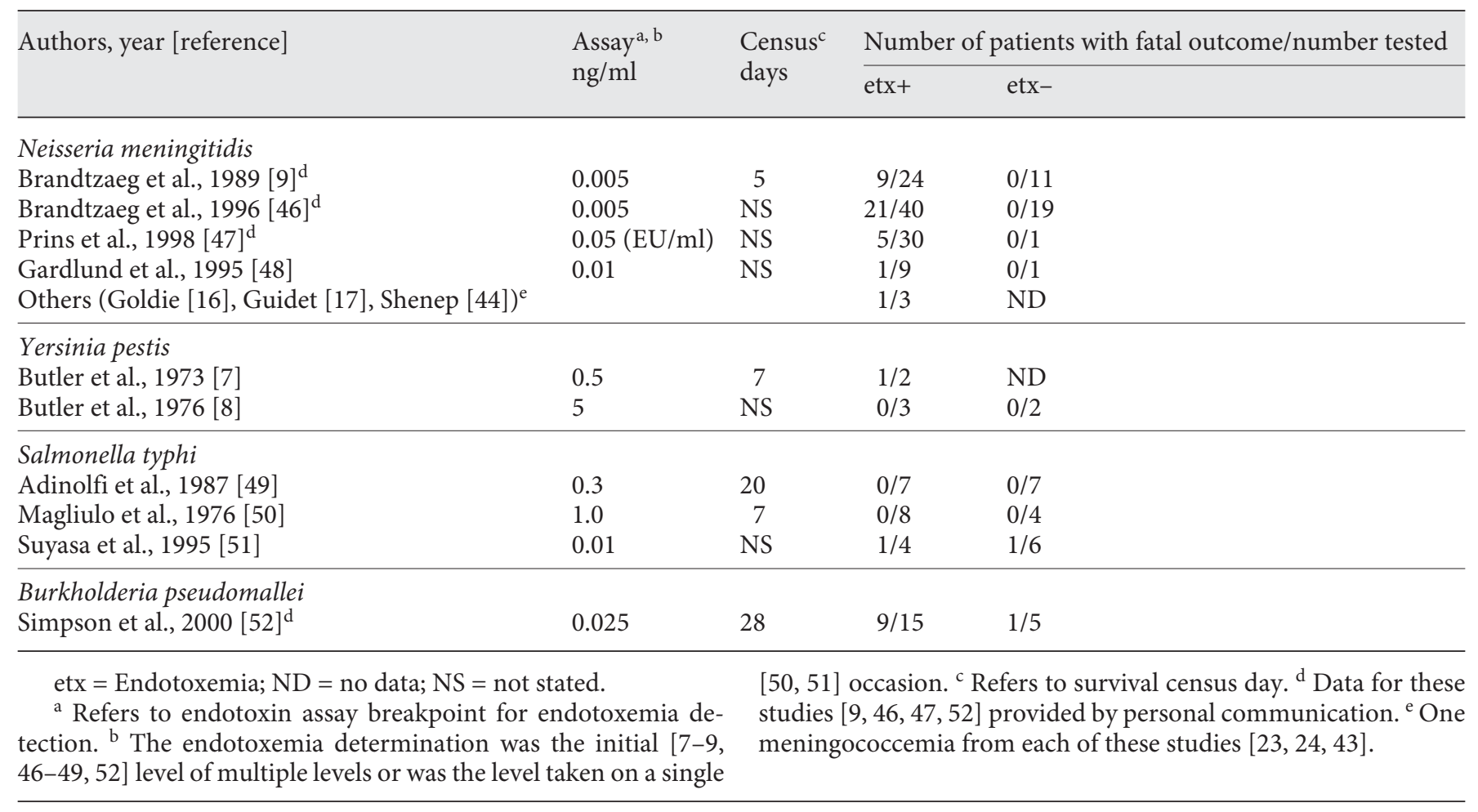

determinations were obtained prior to the administration of antibiotic therapy whereas for the remaining studies this was not stated.

The summary ORs for the three categories of GN bacteremias are summarized in table 3 and presented as a forest plot in figure 1. The largest study [12] provided data in relation to endotoxemia detection at two breakpoints which have been used here to stratify the mortality proportions from this study within figure 1 . None of the study level ORs (fig. 1) were significantly elevated above 1 for any of the three categories of GN bacteremia (table 3 ). The $\mathrm{I}^{2}$ associated with each of these summary ORs was $<10 \%$, indicating minimal heterogeneity (fig. 1 ).

For an analysis with all the unrestricted studies included, only in the case of non-E. coli Enterobacteriaceae was the summary OR significantly elevated above 1, indicative of a worse outcome in endotoxemic patients (table 3 ). The $\mathrm{I}^{2}$ associated with each of these summary ORs was again $<10 \%$ (data not shown). The increased mortality for this category is particularly apparent for Klebsiella species ( 9 of 13 deaths in 8 studies if endotoxemia was detected vs. 2 of 17 deaths in 6 studies if endotoxemia was not detected). The method used to derive the summary ORs in either figure 1 or table 3 made no substantive difference to the overall findings. For an analysis with only the ICU studies included, none of the summary ORs were significantly elevated above 1 .

\section{Enterobacteriaceae Bacteremias and Endotoxemia}

The effect of the detection of endotoxemia on mortality for GN bacteremias with $E$. coli versus Enterobacteriaceae other than E. coli (table 3) was tested as an interaction term within a regression model. This interaction term was found to be significant indicating an impact of endotoxemia detection on prognosis additional to the impact of GN bacteremia type. This interaction term was found to be significant (OR 2.8; 1.01-8.1) for all unrestricted studies (table 3 ) and also for an analysis limited to the studies undertaken in ICU setting (OR 3.8; 1.13-12.8).

\section{Restricted Studies}

There were 10 studies that were restricted to one of the following four GN pathogen types (table 2): Neisseria meningitidis (4 studies [9, 46-48]), Burkholderia pseudomallei (1 study [52]), Yersinia pestis (2 studies [7, 8]), and Salmonella typhi (3 studies [49-51]) in which 201 bacteremic patients experienced 50 (25\%) fatal outcomes. For these GN bacteremias, only in the case of Neisseria men- 
Table 3. Summary mortality proportions and ORs in association with endotoxemia detection

\begin{tabular}{|c|c|c|c|c|c|}
\hline GN bacteremia species & $\begin{array}{l}\text { Number } \\
\text { of studies }\end{array}$ & $\begin{array}{l}\text { endotoxemia } \\
\text { detected }\end{array}$ & $\begin{array}{l}\text { endotoxemia } \\
\text { not detected }\end{array}$ & GEE & ELR \\
\hline \multicolumn{6}{|l|}{ ICU studies only } \\
\hline E. coli $i^{\mathrm{a}}$ & 8 & $12 / 64(19)$ & $15 / 43(35)$ & $0.54 ; 0.25-1.13$ & $0.51 ; 0.17-1.5$ \\
\hline Non-E. coli Enterobacteriaceae ${ }^{\mathrm{a}, \mathrm{b}}$ & 8 & $20 / 50(40)$ & $6 / 25(24)$ & $2.7 ; 0.90-8.4$ & $3.1 ; 0.82-13.7$ \\
\hline \multicolumn{6}{|l|}{ All studies } \\
\hline E. $\operatorname{coli}^{\mathrm{a}}$ & 15 & $17 / 81(21)$ & $17 / 63(27)$ & $0.78 ; 0.36-1.7$ & $0.81 ; 0.28-2.3$ \\
\hline Non-E. coli Enterobacteriaceae ${ }^{\mathrm{a}, \mathrm{b}}$ & 14 & $26 / 62(42)$ & $7 / 35(20)$ & $3.7 ; 1.3-10.3$ & $3.9 ; 1.1-16.7$ \\
\hline Non-Enterobacteriaceae $e^{c}$ & 13 & $22 / 53(42)$ & $8 / 25(32)$ & $1.7 ; 0.65-4.6$ & $1.4 ; 0.42-5.3$ \\
\hline Neisseria meningitides & 4 & $37 / 106(35)$ & $0 / 32(0)$ & $26.0 ; 1.6-321$ & 36.9; 7.7-inf. \\
\hline Yersinia pestis & 2 & $1 / 5(20)$ & $0 / 2(0)$ & undefined & undefined \\
\hline
\end{tabular}

GEE $=$ Generalized estimating equation method; ELR $=$ exact logistic regression method.

a The impact of the detection of endotoxemia on the difference in morality proportions for E. coli versus non-E. coli Enterobacteriaceae bacteremias was tested as an interaction term within a regression model using generalized estimating equations. This interaction term was found to be significant for an analysis in- cluding all studies (OR 2.8; $1.01-8.1$ ) and for an analysis limited to the studies undertaken in ICU only (OR $3.8 ; 1.13-12.8$ ). ${ }^{b}$ Non-E.coli Enterobacteriaceaeincludes thefollowing bacteremias: Klebsiella species, Enterobacter species, Proteus species, and Serratia species. ${ }^{\mathrm{c}}$ Non-Enterobacteriaceae includes the following bacteremias: Pseudomonas species, Acinetobacter species, and Bacteroides species. ingitidis bacteremia was the summary OR for mortality significantly elevated $(26.0 ; 1.6-321 ; \mathrm{p}<0.0001)$ in association with the detection of endotoxemia (table 3 ).

\section{Discussion}

GN bacteremia has a high mortality and observations derived from animal models implicate a key role for endotoxemia in its pathogenesis. From this, it could be hypothesized that the differences in relative frequency of detection of concomitant endotoxemia with GN bacteremia on the one hand $[30,53,54]$, or the known differences in the structure activity relationship of the lipid A component of the endotoxin molecule on the other $[1,22$, 27-28], might account for differences in mortality between bacteremias with Enterobacteriaceae versus other GN bacteria. However, the findings here were unexpectedly otherwise.

This analysis updates two previous meta-analyses [21, 53] applying current analytic methodology [31, 33], using available patient level data to enable questions relating to the interaction between GN bacteremia species type and endotoxemia detection on prognosis to be addressed.
There are several obstacles to resolve in undertaking the analysis presented here. Firstly, the studies were diverse with respect to their dates of publications, sizes, study designs, endotoxemia breakpoint levels, survival census times, patient populations, and settings. By contrast, the studies conducted in the ICU setting exhibited less diversity with respect to these features and were all large and published in the 1990s. Second, the data is sparse, unbalanced and clustered. In undertaking a meta-analysis the Mantel-Haenszel method is commonly used for data analysis and also to provide a graphical summary together with computational tests for heterogeneity. However, this method may be suboptimal for deriving and comparing summaries under the data conditions found here. So two additional analytic methods were employed here. Logistic regression using exact methods and the method of generalized estimating equations are more computationally intensive methods which allow a population-averaged summation under less restrictive assumptions regarding the underlying population distributions and data independence [33].

There are five findings here:

(1) The prognostic value of endotoxemia detection in association with GN bacteremia studies varies across dif- 
Author

E. coli

Goldie [16]

Bates [14]

Strutz [36]

Opal [12] low

Guidet [17]

Opal [12] high

Dofferhoff [34]

Adult non-ICU

Oncology and pediatric

Danner [15]

Subtotal $\left(I^{2}=0.0 \%, p=0.733\right)$

Non-E. coli Enterobacteriaceae

Goldie [16]

Opal [12] low

Opal [12] high

Oncology and pediatric

Bates [14]

Danner [15]

Dofferhoff [34]

Adult non-ICU

Subtotal $\left(I^{2}=0.0 \%, p=0.810\right)$

Non-Enterobacteriaceae

Guidet [17]

Adult non-ICU

Opal [12] high

Opal [12] low

Bates [14]

Goldie [16]

Oncology and pediatric

Subtotal $\left(I^{2}=0.0 \%, p=0.779\right)$

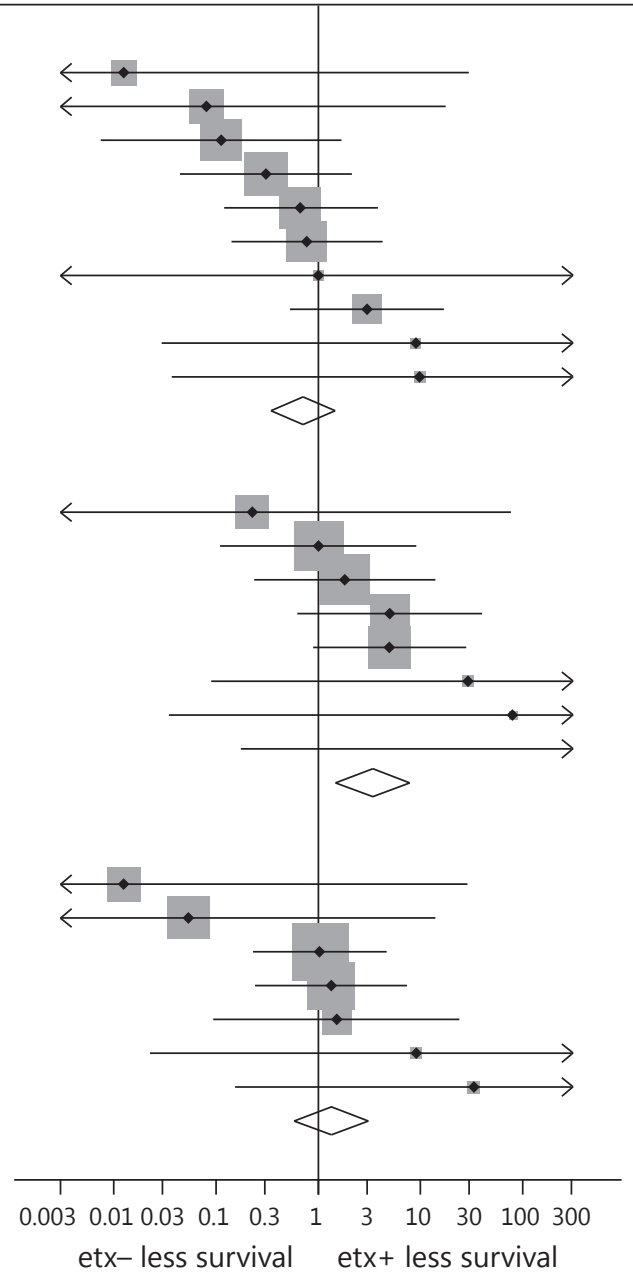

Fig. 1. Forest plot of mortality OR of patients with GN bacteremia in relation to whether endotoxemia was (etx + ) or was not (etx-) detected presented as study specific and summary OR and 95\% CI. 'etx- less survival' and 'etx+ less survival' indicates the respective OR ranges for which mortality was more common in association with either endotoxemia non-detection or detection, respectively. Arrowheads indicate 95\% CIs that extend out of range.

ferent GN bacteremia species. This variability across species types, which is in contrast to the lack of heterogeneity across studies, is visually apparent in the forest plot (fig. 1).

(2) The endotoxemia detection in association with bacteremias with Neisseria meningitidis and with Enterobacteriaceae other than E. coli has a strong prognostic value.

(3) Surprisingly, the detection of endotoxemia in association with E. coli bacteremia, which is the second largest GN bacteremia category here, has no prognostic value. No single study included here would have been large enough to demonstrate this observation. As a sensitiv- ity analysis, it was found that it would require at least three studies each having at least 40 patients with $E$. coli bacteremias with an OR for mortality of $>10$ in association with the detection versus non-detection of endotoxemia to change the conclusions found here (data not shown). It is unlikely that such studies would be unpublished or easily overlooked.

(4) The overall better prognosis associated with E. coli bacteremias [25] versus non-E. coli Enterobacteriaceae (table 3) cannot be attributed to endotoxemia being less frequently detectable among $E$. coli bacteremias. 
(5) For the largest study [12], the 135 GN bacteremic patients were able to be classified into subgroups with high $(>660 \mathrm{pg} / \mathrm{ml})$, low $(25-660 \mathrm{pg} / \mathrm{ml})$ and non-detectable $(<25 \mathrm{pg} / \mathrm{ml})$ levels of endotoxemia. This simple dose-response analysis did not suggest a higher mortality OR with higher levels of endotoxemia in any of the three GN bacteremia categories studied (fig. 1). These findings are particularly surprising given that the lipid A structure of LPS (endotoxin) of Enterobacteriaceae, including E. coli, is typically hexa-acyl, a structure which is known to have a key role toward recognition by the MD2:TLR4 receptor and the stimulation of cytokine release $[1,55]$.

\section{Limitations}

There were small numbers of bacteremias with Yersinia pestis (plague), and Salmonella typhi (typhoid) and the non-Enterobacteriaceae, and within these bacteremia groups there were small numbers with endotoxemia not detected. Hence the estimates of mortality proportions for these categories of GN bacteremias and the impact of endotoxemia detection are least stable to the appearance of additional data.

Many relevant patient-specific details such as age and patient comorbidities were not available. Both endotoxemia and GN bacteremia are episodic phenomena [56] and endotoxemia levels may be increased by antibiotic therapy [57]. For all but three studies the timing of antibiotic administration in relation to these determinations is unclear. Moreover, the breakpoints used to determine a positive endotoxemia result differed across the studies.

The studies examined in this analysis differed from each other in several respects and several study level factors would have potentially varied over the time period over which these studies had been conducted. For example, the antibiotic and supportive therapies, and the blood culture methods for detecting GN bacteremias, have not been considered here. These are all factors that could be expected to have varied over the more than 25 years covered in this overview, particularly with respect to the studies published prior to 1990 .

Despite the diverse mix of study designs and settings, all summary ORs are associated with $\mathrm{I}^{2}$ less than $10 \%$, a finding that indicates minimal heterogeneity. This is a surprising finding given the diversity of patient groups, underlying risk, and clinical settings. Minimal heterogeneity was also noted with the analysis repeated with studies undertaken in non-ICU settings included. The studies undertaken in the ICU setting were of particular interest

Endotoxemia and Gram-Negative

Bacteremia as published benchmarks for the mortality proportions for GN bacteremias in this patient group are available [25]. For example, the median mortality proportion among $>1,000$ bacteremias in ICU patients abstracted from $>7$ large studies is 19\%, $28-30 \%$ and $32 \%$ for bacteremias with E. coli, Enterobacteriaceae other than E. coli, and $P$. aeruginosa, respectively [25] which can be used as literature-derived benchmarks. Among the three GN bacteremia categories noted in the studies included here (table 3 ), the mortality proportions and their relative rankings are similar to these benchmarks [25].

This overview is based on a summation of observational studies. While the mortality risk in association with the detection of endotoxemia is increased for some but not all types of GN bacteremias, this overview is unable to identify the causal mechanism for this increased risk.

An increased OR for mortality in association with the detection of endotoxemia may be due to other associations. For example, the highly significant OR for mortality in association with meningococcemia may be confounded by the quantitative level of bacteremia. Brandtzaeg and colleagues [58] have found that the level of endotoxemia correlates with the level of neisserial DNA in plasma in this patient group. Likewise for other GN bacteremias, the level of bacteremia, as reflected using quantitative blood culture methods [59] or in time to blood culture positivity $[60,61]$, is an important determinant of prognosis.

However, the kinetics of bacteremia, endotoxemia and pathophysiology may not be concordant. For example, in one clinical study [15] of 100 patients with sepsis in an ICU setting, the cumulative percent found to have endotoxemia rose from 20 to $40 \%$ between 0 and $24 \mathrm{~h}$ after study entry. Moreover, there are observations in animal models of septic shock conducted under controlled conditions with quantitative measurements of endotoxemia, GN bacteremia, hemodynamic changes and duration of survival which relate to this non-concordance $[22,23]$. Following intraperitoneal challenge with strains of E. coli which have (O6:H1:K2) or do not have (O86:H8) virulence factors for human disease, survival time was shorter and the associated hemodynamic changes were more severe after challenge with the virulent strain, as might be expected. However, there are three paradoxical observations as follows: (1) bacteremia occurred earlier and more frequently after challenge with the avirulent $E$. coli strain; (2) levels of endotoxemia were three-fold higher after challenge with the avirulent E. coli strain, and (3) challenge with heat-killed bacteria at a ten-fold higher dose 
was associated with a reversal of the effects on survival and hemodynamic change seen with live bacterial challenge: the survival was now significantly shortened after challenge with the killed non-virulent bacteria versus the killed virulent bacteria. Despite this reversal, the levels of endotoxemia were again three-fold higher after challenge with the killed avirulent versus the killed virulent $E$. coli strain $[22,23]$.

The co-presence of endotoxemia and GN bacteremia may also reflect important differences in host factors. In a study of 68 patients with GN bacteremia, Young [62] found a strong association between endotoxemia and recognized adverse prognostic host factors such as leukopenia, low opsonic titers in plasma against the autologous bacteremia strain and more severe underlying disease. Also, in an animal model, it has been noted that endotoxemia impedes the clearance of bacteremia [63].

It is notable that endotoxin derived from $E$. coli is used commonly in animal and preclinical studies [64]. Moreover, future clinical investigations should consider the types and co-detection of GN bacteremia as confounding factors [65] in clinical associations of endotoxemia.

\section{Conclusion}

The impact of co-detection of endotoxemia with GN bacteremia on patient prognosis is unequal for different types of GN bacteremia, even amongst Enterobacteriaceae. This finding is surprising given the commonality of hexa-acyl lipid A structures among the endotoxins of Enterobacteriaceae. In the light of experimental findings, it is plausible that the unexpected clinical observations here may reflect differential kinetics of endotoxemia and GN bacteremia which are consequent on the presence of different virulence factors present in different species of GN bacteria [66].

\section{Acknowledgments}

We thank Dr. P. Brandtzaeg, Ulleval Hospital, Norway; Dr. B. Byl, Erasme University Hospital, Belgium; Dr. P. Engervall, Karolinska Hospital, Sweden; Dr. M. Yoshida, Jichi Medical School, Japan; Dr. D. Bates, Brigham and Women's Hospital, USA; Dr. R. Danner, NIH, Bethesda, USA; Dr. B. Guidet, Hopital Saint-Antoine, France; Dr. E. Maury, Hopital Saint-Antoine, France; Dr. K.C.H. Fearon, Royal Infirmary, UK; Dr. E.J. Giamarellos-Bourboulis, Attikon University Hospital, Greece, and Dr. J. Prins, Academic Medical Center, The Netherlands, for the individual patient data. The manuscript has been submitted in partial fulfilment for the degree of Doctor of Medical Science at The University of Melbourne.

Ethics approval was not required for this study.

\section{Disclosure Statement}

Financial support was provided by the author. Financial disclosures and potential conflicts of interest: The authors have no conflicts to declare. Role of the funder: The funder had no role in the design, analysis or writing of this study or in its submission for publication. The author is independent from the funder.

\section{References}

1 Munford RS: Sensing gram-negative bacterial lipopolysaccharides: a human disease determinant? Infect Immun 2008;76:454-465.

$\checkmark 2$ Hurley JC: Endotoxemia: methods of detection and clinical correlates. Clin Micro Rev 1995;8:268-292.

3 Opal S, Gluck T: Endotoxin as a drug target. Crit Care Med 2003;31:S57-S64.

4 Hurley JC, Guidet B, Offenstadt G, Maury E: Endotoxemia and mortality prediction in ICU and other settings: underlying risk and co-detection of gram negative bacteremia are confounders. Crit Care 2012;16:R148.

5 Dyson A, Singer M: Animal models of sepsis: Why does preclinical efficacy fail to translate to the clinical setting? Crit Care Med 2009; 37:S30-S37.

6 Rittirsch D, Hoesel LM, Ward PA: The disconnect between animal models of sepsis and human sepsis. J Leukoc Biol 2007;81:137-143.
7 Butler T, Levin J, Cu DQ, Walker RI: Bubonic plague: detection of endotoxemia with the limulus test. Ann Intern Med 1973;79:642_646.

8 Butler T, Levin J, Linh NN, Chau DM, Adickman M, Arnold K: Yersinia pestis infection in Vietnam. II. Quantitative blood cultures and detection of endotoxin in the cerebrospinal fluid of patients with meningitis. J Infect Dis 1976;133:493-499.

-9 Brandtzaeg P, Kierulf P, Gaustad P, et al: Plasma endotoxin as a predictor of multiple organ failure and death in systemic meningococcal disease. J Infect Dis 1989;159:195-204.

10 van Deventer SJ, Buller HR, ten Cate JW, Sturk A, Pauw W: Endotoxaemia: an early predictor of septicaemia in febrile patients. Lancet 1988;1:605-609.
11 Levin J, Poore TE, Young NS, et al: Gramnegative sepsis: detection of endotoxemia with the limulus test. With studies of associated changes in blood coagulation, serum lipids and complement. Ann Intern Med 1972; 76:1-7.

12 Opal SM, Scannon PJ, Vincent JL, et al: Relationship between plasma levels of lipopolysaccharide (LPS) and LPS-binding protein in patients with severe sepsis and septic shock. J Infect Dis 1999; 180:1584-1589.

13 Stumacher RJ, Kovnat MJ, McCabe WR: Limitations of the usefulness of the limulus assay for endotoxin. N Engl J Med 1973;288:12611264.

14 Bates DW, Parsonnet J, Ketchum PA, et al: Limulus lysate assay for detection of endotoxin in patients with sepsis syndrome. Clin Infect Dis 1998;27:582-591. 
15 Danner RL, Elin RJ, Hosseini JM, Wesley RA, Reilly JM, Parillo JE: Endotoxemia in human septic shock. Chest 1991;99:169-175.

16 Goldie AS, Fearon KCH, Ross JA, et al: Natural cytokine antagonists and endogenous anti-endotoxin core antibodies in sepsis syndrome. JAMA 1995;274:172-177.

17 Guidet B, Barakett V, Vassal T, Petit JC, Offenstadt G: Endotoxemia and bacteremia in patients with sepsis syndrome in the intensive care unit. Chest 1994;106:1194-1201.

18 Venet C, Zeni F, Viallon A, et al: Endotoxaemia in patients with severe sepsis or septic shock. Intensive Care Med 2000;26:538-544.

19 Kollef MH, Eisenberg PR: A rapid quantitative assay to detect circulating endotoxin can predict the development of multiorgan dysfunction. Chest 1997;112:173-180.

20 Casey LC, Balk RA, Bone RC: Plasma cytokine and endotoxin levels correlate with survival in patients with the sepsis syndrome. Ann Intern Med 1993;119:771-778.

-21 Hurley JC: Endotoxemia and Gram-negative bacteremia as predictors of outcome in sepsis: a meta-analysis using ROC curves. J Endotoxin Res 2003;9:271-279.

-22 Danner RL, Natanson C, Elin RJ, et al: Pseudomonas aeruginosa compared with Escherichia coli produces less endotoxemia but more cardiovascular dysfunction and mortality in a canine model of septic shock. Chest 1990;98:1480-1487.

23 Hoffman WD, Danner RL, Quezado ZM, Banks SM, Elin RJ, Hosseini JM, Natanson C: Role of endotoxemia in cardiovascular dysfunction and lethality: virulent and nonvirulent Escherichia coli challenges in a canine model of septic shock. Infect Immun 1996;64: 406-412.

24 Roberts FJ, Geere IW, Coldman A: A threeyear study of positive blood cultures, with emphasis on prognosis. Rev Infect Dis 1991;13: 34-46.

25 Cohen J, Cristofaro P, Carlet J, Opal S: New method of classifying infections in critically ill patients. Crit Care Med 2001;32:1510-1526.

26 McCabe WR, Jackson GG: Gram negative bacteremia. II Clinical laboratory and therapeutic observations. Arch Intern Med 1962; 110:856-864

27 Dehus O, Hartung T, Hermann C: Endotoxin evaluation of eleven lipopolysaccharides by whole blood assay does not always correlate with Limulus amebocyte lysate assay. J Endotoxin Res 2006;12:171-180.

28 Devleeschouwer MJ, Cornil MF, Dony J: Studies on the sensitivity and specificity of the Limulus amebocyte lysate test and rabbit pyrogen assays. Appl Environ Microbiol 1985; 50:1509-1511.

29 Hurley JC: Does gram negative bacteremia occur without endotoxemia? A meta-analysis using hierarchical summary ROC curves. Eur J Clin Microbiol Infect Dis 2010;29:207-215.

-30 Hurley JC: Endotoxemia and gram-negative bacteremias predictors of outcome in sepsis; a call for data. J Endotox Res 2001;7:467.
31 Harris RJ, Bradburn MJ, Deeks JJ, Harbord RM, Altmamn DG, Sterne JAC: Metan: fixed and random effects-meta-analysis. Stata J 2008;8:3-28.

32 Higgins JP, Thompson SG, Deeks JJ, Altman DG: Measuring inconsistency in meta-analyses. BMJ 2003;327:557-560.

33 Mutapi F, Roddam A: p values for pathogens: statistical inference for infectious-disease data. Lancet Infect Dis 2002;2:219-230.

34 Dofferhoff ASM, Bom VJJ, de Vries-Hospers HG, et al: Patterns of cytokines, plasma endotoxin, plasminogen activator inhibitor, and acute phase proteins during the treatment of severe sepsis in humans. Crit Care Med 1992; 20:185-192.

- 35 Maury E, Barakett V, Blanchard H, Guitton C, Fitting C, Vassal T, Chauvin P, Bertrand Guidet B, Offenstadt G: Circulating endotoxin during initial antibiotic treatment of severe gram-negative bacteremic infections. J Infect Dis 1998; 178:270-273.

36 Strutz F, Heller G, Krasemann K, Krone B, Muller GA: Relationship of antibodies to endotoxin core to mortality in medical patients with sepsis syndrome. Intensive Care Med 1999;25:435-444.

37 Martinez LA, Quintiliani R, Tilton RC: Clinical experience on the detection of endotoxemia with the limulus test. J Infect Dis 1973; 127:102-105.

-38 Byl B, Clevenbergh P, Kentos A, Jacobs F, Marchant A, Vincent JL, Thys JP: Ceftazidime- and imipenem-induced endotoxin release during treatment of gram-negative infections. Eur J Clin Microbiol Infect Dis 2001; 20:804-807.

39 Giamarellou-Bourboulis EJ, Perdios J, Lelekis M, Eoconomou E, Tsouroulas P, Giamarellou $\mathrm{H}$ : Impact of cefuroxime administration on endotoxin (LPS) and tumour necrosis factoralpha (TNFalpha) blood levels in patients suffering from acute pyelonephritis: a preliminary report. Int J Antimicrob Agents 1999;11: 115-119.

40 Prins JM, van Agtmael MA, Kuijper EJ, van Deventer SJ, Speelman P: Antibiotic-induced endotoxin release in patients with gram-negative urosepsis: a double-blind study comparing imipenem and ceftazidime. J Infect Dis 1995; 172:886-891.

41 Hynninen M, Valtonen M, Vaara M, Markkanen H, Kuusela P, Saxen H, Takkunen O: Plasma endotoxin and cytokine levels in neutropenic and non-neutropenic bacteremic patients. Eur J Clin Microbiol Infect Dis 1995; 14:1039-1045.

42 Engervall P, Granstrom M, Andersson B, Kalin M, Bjorkholm M: Endotoxemia in febrile patients with hematological malignancies. Relationship of type of bacteremia, clinical findings and serum cytokine pattern. Infection 1997;25:2-7.
43 Yoshida M, Obayashi T, Tamura H, Tanaka S, Kawai T, Sakamoto S, Miura Y: Diagnostic and prognostic significance of plasma endotoxin determination in febrile patients with haematological malignancies. Eur J Cancer 1994;30A:145-147.

-44 Shenep JL, Flynn PM, Barrett FF, Stidham GL, Westenkirchner DF: Serial quantitation of endotoxemia and bacteremia during therapy for gram-negative bacterial sepsis. J Infect Dis $1988 ; 157: 565-568$.

45 Ahmed T, Azam MA, Armed N, Jamil KM, Hassan F, Ogura N, Tamura H, Yokochi T: Detection of endotoxin in sera from children hospitalized for treatment of diarrhea in Bangladesh. J Endotoxin Res 2004;10:223-228.

46 Brandtzaeg P, Hogasen K, Kierulf P, Mollnes TE: The excessive complement activation in fulminant meningococcal septicemia is predominantly caused by alternative pathway activation. J Infect Dis 1996;173:647-655.

47 Prins JM, Lauw FN, Derkx BH, Speelman P, Kuijper EJ, Dankert J, van Deventer SJ: Endotoxin release and cytokine production in acute and chronic meningococcaemia. Clin Exp Immunol 1998;114:215-219.

-48 Gardlund B, Sjolin J, Nilsson A, Roll M, Wickerts CJ, Wretlind B: Plasma levels of cytokines in primary septic shock in humans: correlation with disease severity. J Infect Dis 1995;172:296-301.

49 Adinolfi LE, Utili R, Gaeta GB, Perna P, Ruggiero G: Presence of endotoxemia and its relationship to liver dysfunction in patients with typhoid fever. Infection 1987;15:359362 .

50 Magliulo E, Scevola D, Fumarola D, Vaccaro R, Bertotto A, Burberi S: Clinical experience in detecting endotoxemia with the limulus test in typhoid fever and other Salmonella infections. Infection 1976;4:21-24.

-51 Suyasa IG, Reka IG, Inada K, Suda H, Kojima M, Mushiaki K, Okamoto S, Yoshida M: Plasma endotoxin in typhoid fever. Kobe J Med Sci 1995;41:175-186.

52 Simpson AJ, Opal SM, Angus BJ, Prins JM, Palardy JE, Parejo NA, Chaowagul W, White NJ: Differential antibiotic-induced endotoxin release in severe melioidosis. J Infect Dis 2000; 181:1014-1019.

53 Hurley JC: Reappraisal with meta-analysis of bacteremia, endotoxemia and mortality in Gram negative sepsis. J Clin Microbiol 1995; 33:1278-1282.

54 Hurley JC: The detection of endotoxemia with Gram negative bacteremia is bacterial species dependent: A meta-analysis of clinical studies. J Clin Microbiol 2009;47:3826-3831.

55 Kim HM, Park BS, Kim J-I, et al: Crystal structure of the TLR4-MD-2 complex with bound endotoxin antagonist eritoran. Cell 2007;130: 906-917.

-56 Klein DJ, Derzko A, Foster D, Seely AJ, Brunet F, Romaschin AD, Marshall JC: Daily variation in endotoxin levels is associated with increased organ failure in critically ill patients. Shock 2007;28:524-529.
Endotoxemia and Gram-Negative Bacteremia
J Innate Immun 2013;5:555-564 DOI: $10.1159 / 000347172$ 
57 Hurley JC: Antibiotic induced release of endotoxin: a reappraisal. Clin Infect Dis 1992; 15:840-854.

58 Øvstebø R, Brandtzaeg P, Brusletto B, Haug KB, Lande K, Høiby EA, Kierulf P: Use of robotized DNA isolation and real-time PCR to quantify and identify close correlation between levels of Neisseria meningitidis DNA and lipopolysaccharides in plasma and cerebrospinal fluid from patients with systemic meningococcal disease. J Clin Microbiol 2004;42:2980-2987.

59 Kreger BE, Craven DE, Carling PC, McCabe WR: Gram-negative bacteremia. III. Reassessment of etiology, epidemiology and ecology in 612 patients. Am J Med 1980;68:332343
60 Peralta G, Roiz MP, Sanchez MB, Garrido JC, Ceballos B, Rodriguez-Lera MJ, Mateos F, De Benito I: Time-to-positivity in patients with Escherichia coli bacteraemia. Clin Microbiol Infect 2007;13:1077-1082.

61 Liao CH, Lai CC, Hsu MS, et al: Correlation between time to positivity of blood cultures with clinical presentation and outcomes in patients with Klebsiella pneumoniae bacteraemia: prospective cohort study. Clin Microbiol Infect 2009; 15:1119-1125.

62 Young LS: Opsonizing antibodies, host factors, and the limulus assay for endotoxin. Infect Immun 1975;12:88-92.
63 Koch T, Duncker HP, Axt R, Schiefer GH, Van Ackern K, Neuhof H: Alterations of bacterial clearance induced by endotoxin and tumor necrosis factor. Infect Immun 1993;61: 3143-3148.

64 Cross AS, Opal SM, Sadoff JC, Gemski P: Choice of bacteria in animal models of sepsis. Infect Immun 1993;61:2741-2747.

65 Miller PJ, Wenzel RP: Etiologic organisms as independent predictors of death and morbidity associated with bloodstream infections. J Infect Dis 1987;156:471-477.

66 Hurley JC: Reappraisal of the role of endotoxin in the sepsis syndrome. Lancet 1993; 341:1133-1135. 